Article

\title{
An Examination of the Neutralization of In Vitro Toxicity of Chinese Cobra (Naja atra) Venom by Different Antivenoms
}

\author{
Qing Liang ${ }^{1,2}$, Tam Minh Huynh ${ }^{1}$, Nicki Konstantakopoulos ${ }^{1}$, Geoffrey K. Isbister ${ }^{1,3}$ [D \\ and Wayne C. Hodgson ${ }^{1, *}$ \\ 1 Monash Venom Group, Department of Pharmacology, Biomedical Discovery Institute, Monash University, \\ Clayton 3800, Australia; qing.liang@monash.edu (Q.L.); Tommy.Huynh@monash.edu (T.M.H.); \\ Nicki.Konstantakopoulos@monash.edu (N.K.); geoff.isbister@gmail.com (G.K.I.) \\ 2 Department of Emergency Medicine, The First Affiliated Hospital of Guangzhou Medical University, \\ 151 Yanjiang Rd, Guangzhou 510120, China \\ 3 Clinical Toxicology Research Group, University of Newcastle, Callaghan 2308, Australia \\ * Correspondence: wayne.hodgson@monash.edu
}

Received: 26 August 2020; Accepted: 23 September 2020; Published: 25 September 2020

check for updates

\begin{abstract}
The Chinese Cobra (Naja atra) is an elapid snake of major medical importance in southern China. We describe the in vitro neurotoxic, myotoxic, and cytotoxic effects of N. atra venom, as well as examining the efficacy of three Chinese monovalent antivenoms (N. atra antivenom, Gloydius brevicaudus antivenom and Deinagkistrodon acutus antivenom) and an Australian polyvalent snake antivenom. In the chick biventer cervicis nerve-muscle preparation, N. atra venom $(1-10 \mu \mathrm{g} / \mathrm{mL})$ abolished indirect twitches in a concentration-dependent manner, as well as abolishing contractile responses to exogenous acetylcholine chloride (ACh) and carbamylcholine chloride (CCh), indicative of post-synaptic neurotoxicity. Contractile responses to potassium chloride $(\mathrm{KCl})$ were also significantly inhibited by venom indicating myotoxicity. The prior addition of Chinese N. atra antivenom $(0.75 \mathrm{U} / \mathrm{mL})$ or Australian polyvalent snake antivenom $(3 \mathrm{U} / \mathrm{mL})$, markedly attenuated the neurotoxic actions of venom $(3 \mu \mathrm{g} / \mathrm{mL})$ and prevented the inhibition of contractile responses to $\mathrm{ACh}, \mathrm{CCh}$, and $\mathrm{KCl}$. The addition of Chinese antivenom $(0.75 \mathrm{U} / \mathrm{mL})$ or Australian polyvalent antivenom $(3 \mathrm{U} / \mathrm{mL})$ at the $t_{90}$ time point after the addition of venom $(3 \mu \mathrm{g} / \mathrm{mL})$, partially reversed the inhibition of twitches and significantly reversed the venom-induced inhibition of responses to ACh and CCh, but had no significant effect on the response to $\mathrm{KCl}$. Venom $(30 \mu \mathrm{g} / \mathrm{mL})$ also abolished direct twitches in the chick biventer cervicis nerve-muscle preparation and caused a significant increase in baseline tension, further indicative of myotoxicity. $N$. atra antivenom $(4 \mathrm{U} / \mathrm{mL})$ prevented the myotoxic effects of venom $(30 \mu \mathrm{g} / \mathrm{mL})$. However, G. brevicaudus antivenom $(24 \mathrm{U} / \mathrm{mL})$, D. acutus antivenom $(8 \mathrm{U} / \mathrm{mL})$ and Australian polyvalent snake antivenom $(33 \mathrm{U} / \mathrm{mL})$ were unable to prevent venom $(30 \mu \mathrm{g} / \mathrm{mL})$ induced myotoxicity. In the L6 rat skeletal muscle myoblast cell line, N. atra venom caused concentration-dependent inhibition of cell viability, with a half maximal inhibitory concentration $\left(\mathrm{IC}_{50}\right)$ of $2.8 \pm 0.48 \mu \mathrm{g} / \mathrm{mL}$. N. atra antivenom significantly attenuated the cytotoxic effect of the venom, whereas Australian polyvalent snake antivenom was less effective but still attenuated the cytotoxic effects at lower venom concentrations. Neither G. brevicaudus antivenom or D. acutus antivenom were able to prevent the cytotoxicity. This study indicates that Chinese N. atra monovalent antivenom is efficacious against the neurotoxic, myotoxic and cytotoxic effects of $N$. atra venom but the clinical effectiveness of the antivenom is likely to be diminished, even if given early after envenoming. The use of Chinese viper antivenoms (i.e., G. brevicaudus and D. acutus antivenoms) in cases of envenoming by the Chinese cobra is not supported by the results of the current study.
\end{abstract}

Keywords: Naja atra; neurotoxicity; myotoxicity; venom; antivenom; snake 


\section{Introduction}

There are approximately 205 species of snakes in China, of which more than 50 species are venomous [1]. The Chinese Cobra (Naja atra) is one of the top ten most venomous and clinically important species in China [2]. In China, N. atra is mainly distributed south of the Yangtze River, but is also found in Laos and Vietnam. Based on venomic data, Chinese N. atra venom contains a range of toxins, with cardiotoxins and short-chain neurotoxins being the most abundant components [3-6]. We have previously isolated a short-chain neurotoxin, $\alpha$-Elapitoxin-Na1a, from Chinese N. atra venom [7]. However, it has been previously shown that short-chain neurotoxins dissociate readily from human nicotinic acetylcholine receptors (nAChRs) and are unlikely to contribute substantially to neurotoxicity in humans $[7,8]$. The major outcomes of envenoming by Chinese $N$. atra include severe wound necrosis or chronic necrotic ulceration for which large doses of antivenom are administered. Treatment also requires wound infection control and repeated surgical debridement, with the potential for the eventual amputation of limbs. However, marked neurotoxicity including respiratory muscle paralysis is relatively rare [2,9-11].

Antivenoms form the mainstay treatment of systemic snake envenoming. Currently available antivenoms in China include a monovalent N. atra antivenom, and a bivalent elapid (N. atra and Bungarus multicinctus) antivenom in Taiwan [2,10]. However, there are a lack of animal studies or clinical trials that demonstrate the efficacy of $N$. atra antivenom. Unfortunately, the use of non-specific antivenoms is common in mainland China given there are only monovalent snake antivenoms available, i.e., two for elapids: N. atra (Chinese Cobra) antivenom and Bungarus multicinctus (Chinese Krait) antivenom; and two for vipers: Gloydius brevicaudus (Short-Tailed Mamushi) antivenom and Deinagkistrodon acutus (Sharp-nosed Pit Viper) antivenom. G. brevicaudus or D. acutus antivenoms are advocated for the treatment of local necrosis in patients envenomed by N. atra, when specific antivenom is unavailable. However, there is no evidence for the cross-neutralizing ability of these antivenoms for myotoxicity or cytotoxicity, although patients envenomed by these vipers may also experience local necrosis in severe cases [2].

In this study, we examined the in vitro neurotoxic, myotoxic and cytotoxic effects of Chinese N. atra venom and evaluated the efficacy of Chinese N. atra monovalent antivenom in comparison to a polyvalent elapid antivenom (i.e., Australian polyvalent antivenom) and the possible protective effects of Chinese G. brevicaudus and D. acutus antivenoms against the myotoxicity and cytotoxicity induced by N. atra venom.

\section{Experimental Section}

\subsection{Venom and Antivenoms}

Freeze-dried N. atra venom was obtained from Orientoxin Co., Ltd. (Laiyang, Shandong, China). Chinese N. atra monovalent antivenom (Batch number: 20181202; expiry date: 27/12/2021), Chinese G. brevicaudus monovalent antivenom (Batch number: 20190605; expiry date: 18/06/2022), Chinese D. acutus monovalent antivenom (Batch number: 20190101; expiry date: 21/01/2022) were purchased from Shanghai Serum Biological Technology Co., Ltd. (Shanghai, China). Australian polyvalent snake antivenom (Batch number: 055517501; expiry date: 04/2013) was purchased from Seqirus (Melbourne, Australia). The amount of each antivenom required to neutralize in vitro neurotoxicity was based on the quantity of venom in the organ bath. While for the myotoxicity study, in order to achieve a sufficiently high concentration of antivenom for the venom, all antivenoms were tested at $40 \mu \mathrm{L} / \mathrm{mL}$. According to the manufacturer's instructions: $125 \mathrm{U}$ of $N$. atra antivenom neutralizes $1 \mathrm{mg}$ of $N$. atra venom; $1500 \mathrm{U}$ of G. brevicaudus antivenom neutralizes 1-1.25 $\mathrm{mg}$ of G. brevicaudus venom; and $136 \mathrm{U}$ of D. acutus antivenom neutralizes 1-3 mg of D. acutus venom. For the Australian polyvalent antivenom, $1 \mathrm{U}$ of antivenom neutralizes $10 \mu \mathrm{g}$ of venom from the species of snake against which the antivenom is raised (i.e., brown snake, death adder, mulga snake, taipan, tiger snake). 


\subsection{Chemicals and Reagents}

The following chemicals and drugs were used: acetylcholine chloride (ACh; Sigma-Aldrich, St. Louis, MO, USA), carbamylcholine chloride (CCh; Sigma-Aldrich, St. Louis, MO, USA), d-tubocurarine chloride (d-TC; Sigma-Aldrich, St. Louis, MO, USA), potassium chloride (KCl, Ajax Finechem Pty. Ltd., Taren Point, Australia), bovine serum albumin (BSA; Sigma-Aldrich, St. Louis, MO, USA), 0.5\% Trypsin-EDTA (Gibco Thermofisher, Melbourne, Australia), Penicillin/Streptomycin, Dulbecco's Phosphate Buffered Saline, Dulbecco's Modified Eagle Medium (DMEM) GlutaMAX TM, DMSO (Merck; Darmstach, Germany), CellTire 96 Aqueous One Solution Cell Proliferation Assay (MTS assay; Promega; Melbourne, Australia). All chemicals were dissolved or diluted in Milli-Q water unless otherwise stated.

\subsection{Chick Biventer Cervicis Nerve-Muscle Preparation}

Chickens (male; aged 4-10 days) were killed by exsanguination following $\mathrm{CO}_{2}$ inhalation. Two biventer cervicis nerve-muscle preparations were dissected from each chick and mounted in separate organ baths on wire tissue holders under $1 \mathrm{~g}$ resting tension. Preparations were maintained at $34{ }^{\circ} \mathrm{C}$, bubbled with $95 \% \mathrm{O}_{2}$ and $5 \% \mathrm{CO}_{2}$, in $5 \mathrm{~mL}$ organ baths filled with physiological salt solution consisting of (in mM): $118.4 \mathrm{NaCl}, 4.7 \mathrm{KCl}, 1.2 \mathrm{MgSO}_{4}, 1.2 \mathrm{KH}_{2} \mathrm{PO}_{4}, 2.5 \mathrm{CaCl}_{2}, 25 \mathrm{NaHCO}_{3}$, and 11.1 glucose. Venom was dissolved in $0.05 \%(w / v)$ bovine serum albumin (BSA).

For neurotoxicity experiments, indirect twitches were evoked by stimulating the motor nerve at supramaximal voltage $(0.1 \mathrm{~Hz} ; 0.2 \mathrm{~ms} ; 10-20 \mathrm{~V})$ via an electronic stimulator. d-TC $(10 \mu \mathrm{M})$ was then added to the preparations with the subsequent abolishment of twitches indicating that they were nerve-mediated. The twitches were then restored by washing the preparation with physiological salt solution. Electrical stimulation was stopped and contractile responses to exogenous ACh (1 mM for $30 \mathrm{~s}), \mathrm{CCh}(20 \mu \mathrm{M}$ for $60 \mathrm{~s})$, and $\mathrm{KCl}(40 \mathrm{mM}$ for $30 \mathrm{~s})$ obtained. Electrical stimulation was then recommenced for at least $30 \mathrm{~min}$ before the addition of venom or antivenom. To examine the efficacy of antivenom to prevent venom-induced neurotoxicity, antivenom was added to the tissues $10 \mathrm{~min}$ before venom. To examine the efficacy of antivenom to reverse venom-induced neurotoxicity, antivenom was added to the tissues at the $t_{90}$ time point (i.e., when the twitch height was inhibited by $90 \%$ ). At the conclusion of each experiment, $\mathrm{ACh}, \mathrm{CCh}$, and $\mathrm{KCl}$ were re-added as above.

For myotoxicity experiments, the biventer cervicis muscle was directly stimulated $(0.1 \mathrm{~Hz} ; 2 \mathrm{~ms})$ at supramaximal voltage (20-30 V). In these experiments the electrode was placed around the belly of the muscle and d-TC $(10 \mu \mathrm{M})$ remained in the organ bath for the duration of the experiment. Venom was left in contact with the preparation until twitch blockade occurred, or for a maximum $3 \mathrm{~h}$ period. Venom was considered to be myotoxic if it inhibited twitches elicited by direct stimulation and/or caused a contracture of the skeletal muscle (i.e., increase in the baseline tension of the muscle). To examine the ability of antivenom to neutralize venom-induced myotoxicity (i.e., myotoxicity prevention study), tissues were equilibrated with antivenom for $10 \mathrm{~min}$ before venom was added.

Twitch responses and responses to exogenous agonists were measured via a Grass FT03 force displacement transducer and recorded on a PowerLab system (ADInstruments Pty Ltd., Bella Vista, Australia). Animal experiments were approved on 12 May 2017 by the Monash University Ethics Committee application MARP/2017/147. All experiments were performed in accordance with relevant guidelines and regulations.

\subsection{Cell Culture Experiments}

\subsubsection{Venom}

Freeze-dried venom was reconstituted in distilled water on the day of use. Protein content was determined utilizing a BCA protein assay kit according to the manufacturer's instructions. Briefly, venom $(25 \mu \mathrm{L})$ was added in triplicate to a 96-well micro-titer plate. BSA solutions, diluted from $1-0.025 \mathrm{mg} / \mathrm{mL}$, were used as reference standards and distilled water was used as the blank. Absorbance 
was measured at $562 \mathrm{~nm}$ utilizing VERSAmax tunable microplate reader (Molecular Devices, San Jose, CA, USA). Venom stock solutions were stored at $4{ }^{\circ} \mathrm{C}$ until required.

\subsubsection{Heat Inactivation of Fetal Bovine Serum (hiFBS)}

Fetal bovine serum was heated to $56{ }^{\circ} \mathrm{C}$ for $30 \mathrm{~min}$. Following heat-inactivation, serum was sterilized using a $0.22 \mu \mathrm{M}$ Millipore filter (Sigma-Aldrch, North Ryde, Australia). Serum was dispensed into sterile centrifuge tubes and stored at $-20^{\circ} \mathrm{C}$.

\subsubsection{Cells}

The rat skeletal muscle myoblast cell line, L6, was purchased from The American Type Culture Collection (ATCC, Manassas, VA, USA). L6 cells were grown in $175 \mathrm{~cm}^{2}$ flasks (Nunc, Thermofisher, Melbourne, Australia) in culture media DMEM supplemented with 10\% hiFBS and $1 \%$ penicillin/streptomycin (10\% DMEM). Flasks were maintained at $37^{\circ} \mathrm{C}$ with $5 \% \mathrm{CO}_{2}$ and media was replenished every subsequent day. When the cells reached $80 \%$ confluence (assessed by eye using a light microscope), trypsin was then used to lift the cells. Cells were centrifuged and the cell pellet was re-suspended in culture media $(35 \mathrm{~mL})$. Cell suspension $(100 \mu \mathrm{L} /$ well $)$ was aliquoted into four 96 -well cell culture plates (92 wells/plate) (Nunc, Thermofisher, Melbourne, Australia). Plates were maintained at $37^{\circ} \mathrm{C}$ in an atmosphere of $5 \% \mathrm{CO}_{2}$. Media was replenished every second day until cells reached $90 \%$ confluence. For cell differentiation to occur (i.e., skeletal myoblast cells into skeletal myocytes), $10 \%$ DMEM was removed from wells and replaced with DMEM media supplemented with $2 \%$ hiFBS and $1 \%$ penicillin/streptomycin (2\% DMEM). Plates were subsequently maintained at $37^{\circ} \mathrm{C}$ in an atmosphere of $5 \% \mathrm{CO}_{2}$. Media was replenished every second day, for one week, until cell differentiation (i.e., appearance of long striated cells, assessed by eye using a light microscope) was observed.

To maintain L6 stock, cells at passage 2 were lifted using trypsin and centrifuged. Supernatant was discarded and cell pellets were re-suspended in DMEM $(20 \mathrm{~mL})$ supplemented with $30 \%$ hiFBS and $10 \%$ DMSO. Cell suspension was aliquoted into individual $1 \mathrm{~mL}$ cryovials and stored in liquid nitrogen until required. Cells were passaged up to passage 12 before being discarded and a new vial of cells thawed.

\subsubsection{Cell Proliferation Assay (MTS Assay)}

For cell viability experiments, media were removed from wells of differentiated L6 cell culture plates and the wells were washed once with pre-warmed PBS. Venom stock solution was diluted in $2 \%$ DMEM culture media to a final concentration of $100 \mu \mathrm{g} / \mathrm{mL}$. This was subsequently serially diluted either 1.5-fold (100-0.016 $\mu \mathrm{g} / \mathrm{mL})$ or 1.3-fold $(100-0.24 \mu \mathrm{g} / \mathrm{mL})$. Dilutions $(100 \mu \mathrm{L} /$ well $)$ were added in quadruplicate to wells in a cell culture plate. Culture media controls (i.e., cells and media with no venom) and media blanks (i.e., no cells) were also run in parallel. The plates were maintained at $37^{\circ} \mathrm{C}$ with $5 \% \mathrm{CO}_{2}$ for $24 \mathrm{~h}$. Cell culture plates were subsequently removed from the incubator and washed with pre-warmed PBS three times. DMEM culture media $(2 \% ; 50 \mu \mathrm{L} /$ well $)$ and MTS solution $\left(10 \mu \mathrm{L} /\right.$ well) were pre-mixed, and $60 \mu \mathrm{L}$ added to each well. Plates were further incubated at $37^{\circ} \mathrm{C}$ with $5 \% \mathrm{CO}_{2}$ for $1 \mathrm{~h}$. Absorbance was measured at $492 \mathrm{~nm}$ utilizing a VERSAmax tunable microplate reader (Molecular Devices, San Jose, CA, USA).

\subsubsection{Examining the Efficacy of Antivenom}

Media was removed from wells of L6 cell culture plates and the wells were washed once with pre-warmed PBS. Venom stock solutions were diluted to a concentration of 0 (no venom), 2.5 ( $\mathrm{IC}_{50}$ concentration range), 5 (twice $\mathrm{IC}_{50}$ ), 10 (initial concentration where $100 \%$ cell death occurs), or $30 \mu \mathrm{g} / \mathrm{mL}$ (concentration used in myotoxic study) in $\%$ DMEM culture media containing either no antivenom (venom only) or supplemented with $N$. atra monovalent antivenom $(200 \mu \mathrm{L} ; 4 \mathrm{U} / \mathrm{mL})$ Australian polyvalent snake antivenom $(200 \mu \mathrm{L} ; 33 \mathrm{U} / \mathrm{mL})$, G. brevicaudus antivenom $(200 \mu \mathrm{L} ; 24 \mathrm{U} / \mathrm{mL})$, or D. acutus antivenom $(200 \mu \mathrm{L} ; 8 \mathrm{U} / \mathrm{mL})$. 
Each of the dilutions were added in triplicate to L6 culture plates and incubated at $37^{\circ} \mathrm{C}$ with $5 \% \mathrm{CO}_{2}$ for $24 \mathrm{~h}$. Culture plates were removed from the incubator and washed with pre-warmed PBS three times. Fresh DMEM culture media $(50 \mu \mathrm{L} /$ well $)$ and MTS solution $(10 \mu \mathrm{L} /$ well $)$ were pre-mixed, and $60 \mu \mathrm{L} /$ well was added to each well. The plates were further incubated at $37^{\circ} \mathrm{C}$ with $5 \%$ $\mathrm{CO}_{2}$ for $1 \mathrm{~h}$. Absorbance was measured at $492 \mathrm{~nm}$ utilizing VERSAmax tunable microplate reader (Molecular Devices, San Jose, CA, USA).

\subsection{Data Analysis}

For both in vitro neurotoxicity and myotoxicity experiments, twitch height in the chick biventer preparation was measured at regular time intervals and expressed as a percentage of the pre-venom twitch height. In neurotoxicity studies, the time taken for $90 \%$ inhibition of the twitch response ( $t_{90}$ value) was used to determine the potency of N. atra venom. Post-venom contractile responses to $\mathrm{ACh}, \mathrm{CCh}$, and $\mathrm{KCl}$ were expressed as a percentage of their original responses. In myotoxicity studies, the change in gram (g) of muscle baseline tension was measured every $10 \mathrm{~min}$ after venom addition. The maximum change in tension $(\mathrm{g})$ and time $(\mathrm{min})$ to achieve the maximum change in tension were also measured. Comparison of the effects of $N$. atra venom on twitch height, baseline tension, or time to reach maximum change in tension were made using a one-way analysis of variance (ANOVA). Comparison of responses to exogenous agonists before and after the addition of venom or vehicle was made using a Student's paired $t$-test. All ANOVAs were followed by a Bonferroni's multiple comparison post-hoc test. Data are presented as mean \pm standard error of the mean (SEM) of $n$ experiments. All data and statistical analyses were performed using PRISM 8.0.2 (GraphPad Software, San Diego, CA, USA, 2019).

For cell experiments, sigmoidal growth curves were graphed using Prism 8.0.2 as cell viability (\% of maximum) versus log concentration of venom, and $\mathrm{IC}_{50}$ concentrations determined. Bar graphs displaying the efficacy of antivenoms were plotted as a percentage of cell viability. Cell viability was compared in the presence and absence of antivenom using a one-way ANOVA, with Bonferroni's multiple comparisons test. For all statistical tests, $p<0.05$ was considered statistically significant. Data are presented as mean \pm standard error of the mean (SEM) of $n$ experiments.

\section{Results}

\subsection{In Vitro Neurotoxicity}

\subsubsection{Concentration-Dependent Inhibition of Twitches and Exogenous Agonists Responses}

$N$. atra venom $(1-10 \mu \mathrm{g} / \mathrm{mL})$ caused concentration-dependent inhibition of indirect twitches of the chick biventer preparation, when compared to vehicle control $(\mathrm{n}=6$; one-way ANOVA, $p<0.05$; Figure 1a). The potency of the neurotoxic effect of venom was determined by calculating $t_{90}$ or $t_{50}$ (i.e., if the twitch height to decrease by $90 \%$ or $50 \%$, respectively) with values as follows: $1 \mu \mathrm{g} / \mathrm{mL}$ ( $t_{50} 36 \pm 2 \mathrm{~min}$ ), $3 \mu \mathrm{g} / \mathrm{mL}$ ( $\mathrm{t}_{90} 43 \pm 5 \mathrm{~min}$ ), $10 \mu \mathrm{g} / \mathrm{mL}$ ( $\mathrm{t}_{90} 17 \pm 1 \mathrm{~min}$ ). Venom $(1-10 \mu \mathrm{g} / \mathrm{mL}$ ) also abolished contractile responses to exogenous ACh $(1 \mathrm{mM})$ and CCh $(20 \mu \mathrm{M})$, indicating an action at the post-synaptic nerve terminal, and significantly inhibited responses to $\mathrm{KCl}(40 \mathrm{mM})$, indicative of myotoxicity (Figure 1b). 
(a)

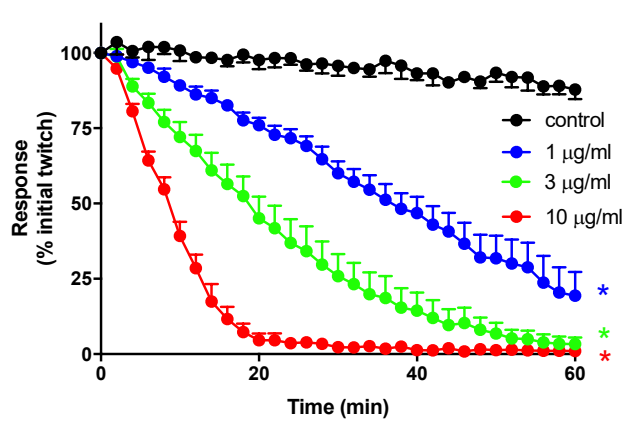

(b)

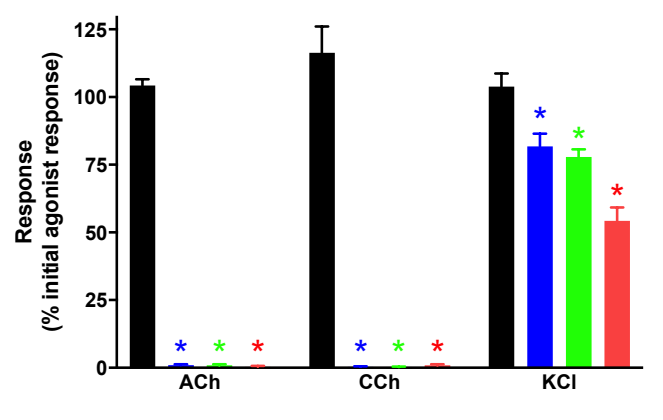

Figure 1. (a) The concentration-dependent neurotoxic effects of $N$. atra venom $(1-10 \mu \mathrm{g} / \mathrm{mL})$ on indirect twitches of the chick biventer cervicis nerve-muscle (CBCNM) preparation. (b) The concentration-dependent effects of $N$. atra venom $(1-10 \mu \mathrm{g} / \mathrm{mL})$ on contractile responses to acetylcholine chloride (ACh) $(1 \mathrm{mM})$, carbachol (CCh) $(20 \mu \mathrm{M})$, and potassium chloride $(\mathrm{KCl})(40 \mathrm{mM})$ in the CBCNM. ${ }^{*} p<0.05$, significantly different from (a) control at $60 \mathrm{~min}$ or (b) pre-venom response to same agonist. $\mathrm{n}=6$.

\subsubsection{In Vitro Neurotoxicity Antivenom Prevention Study}

The prior addition of Chinese $N$. atra monovalent antivenom $(0.75 \mathrm{U} / \mathrm{mL}, 2 \times$ the recommended titre), or Australian polyvalent snake antivenom $(3 \mathrm{U} / \mathrm{mL}, 10 \times$ the recommended titre), markedly attenuated the neurotoxic actions of venom $(3 \mu \mathrm{g} / \mathrm{mL})($ Figure $2 \mathrm{a}, \mathrm{c})$ and prevented the inhibition of contractile responses to $\mathrm{ACh}, \mathrm{CCh}$, and $\mathrm{KCl}$ (Figure 2b,d).
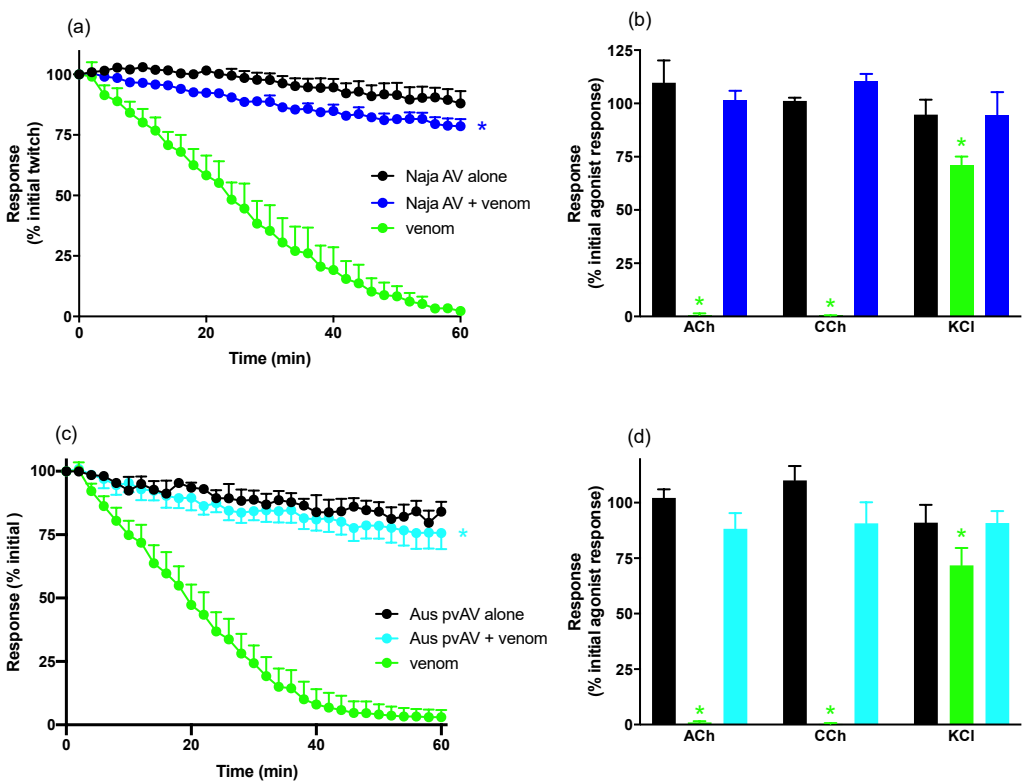

Figure 2. (a) The effects of $N$. atra venom $(3 \mu \mathrm{g} / \mathrm{mL})$ alone or with pre-addition of Naja atra antivenom (Naja AV; $0.75 \mathrm{U} / \mathrm{mL}$ ) on indirect twitches of the CBCNM. (b) The effects of $N$. atra venom $(3 \mu \mathrm{g} / \mathrm{mL})$ alone or with pre-addition of Naja $\mathrm{AV}(0.75 \mathrm{U} / \mathrm{mL})$ on contractile responses to $\mathrm{ACh}(1 \mathrm{mM}), \mathrm{CCh}(20 \mu \mathrm{M})$, and $\mathrm{KCl}(40 \mathrm{mM})$ in the CBCNM. (c) The effects of $N$. atra venom $(3 \mu \mathrm{g} / \mathrm{mL})$ alone or with pre-addition of Australian polyvalent antivenom (Aus pvAV; $3 \mathrm{U} / \mathrm{mL}$ ) on indirect twitches of the CBCNM. (d) The effects of $N$. atra venom $(3 \mu \mathrm{g} / \mathrm{mL})$ alone or with pre-addition of Aus pvAV (3 U/mL) on contractile responses to $\mathrm{ACh}(1 \mathrm{mM}), \mathrm{CCh}(20 \mu \mathrm{M})$, and $\mathrm{KCl}(40 \mathrm{mM})$ in the CBCNM. ${ }^{*} p<0.05$, significantly different compared to venom in the absence of antivenom at $60 \mathrm{~min}(\mathbf{a}, \mathbf{c})$ or compared to pre-venom response to same agonist $(\mathbf{b}, \mathbf{d}) . \mathrm{n}=5-6$. 


\subsubsection{In Vitro Neurotoxicity Antivenom Reversal Study}

The addition of Chinese $N$. atra antivenom $\left(0.75 \mathrm{U} / \mathrm{mL}, 2 \times\right.$ the recommended titre), at the $t_{90}$ time point, after the addition of $N$. atra venom $(3 \mu \mathrm{g} / \mathrm{mL})$, partially restored the twitch height, i.e., reaching $42 \pm 5 \%(n=6)$ of the initial pre-venom twitch height (Figure 3a). Chinese N. atra antivenom also significantly reversed the venom-induced inhibition of responses to $\mathrm{ACh}$ and $\mathrm{CCh}$, while having no significant effect on the response to $\mathrm{KCl}$ (Figure 3b).

(a)

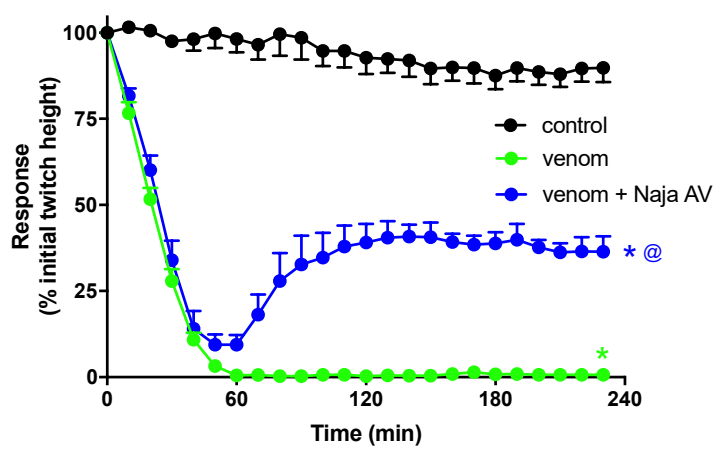

(c)

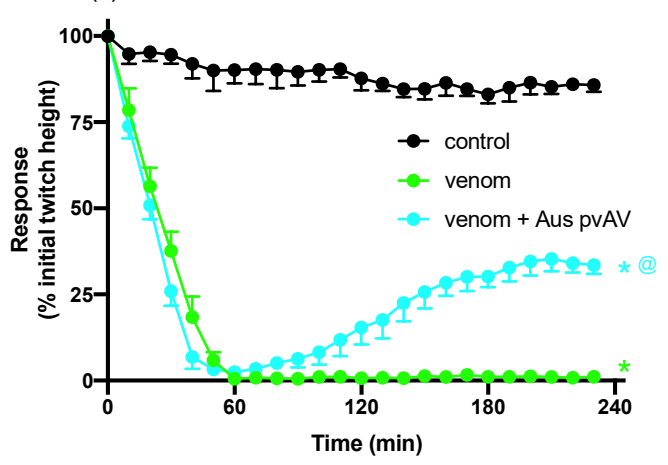

(b)

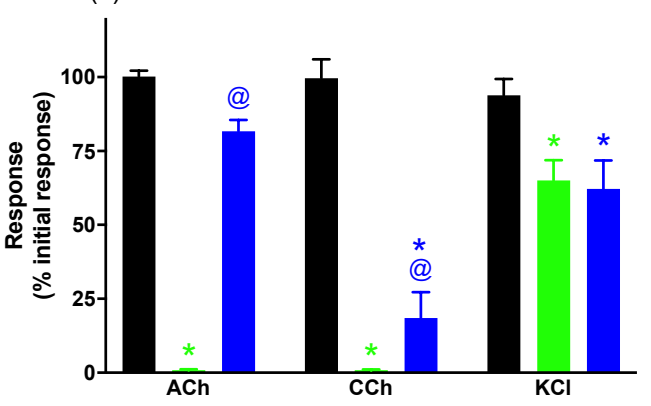

(d)

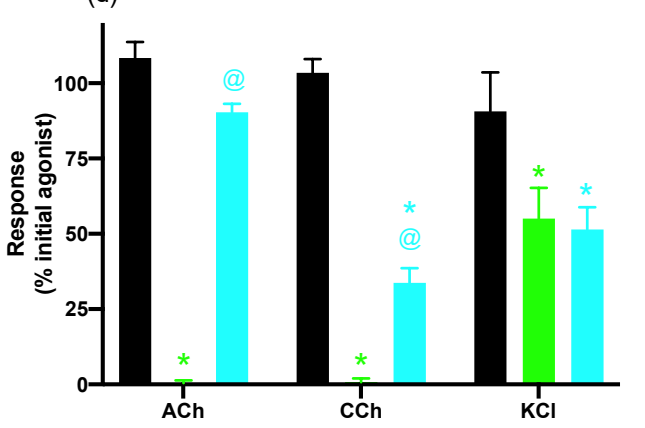

Figure 3. (a) The effects of N. atra venom $(3 \mu \mathrm{g} / \mathrm{mL})$ alone or with Naja atra antivenom (Naja AV; $0.75 \mathrm{U} / \mathrm{mL}$ ) added at the $t_{90}$ time point on indirect twitches of the CBCNM. (b) The effects of N. atra venom $(3 \mu \mathrm{g} / \mathrm{mL})$ alone or with Naja $\mathrm{AV}(0.75 \mathrm{U} / \mathrm{mL})$ added at the $t_{90}$ time point on contractile responses to ACh $(1 \mathrm{mM}), \mathrm{CCh}(20 \mu \mathrm{M})$, and $\mathrm{KCl}(40 \mathrm{mM})$ in the CBCNM. (c) The effects of $N$. atra venom $\left(3 \mu \mathrm{g} / \mathrm{mL}\right.$ ) alone or with Australian polyvalent antivenom (Aus pvAV; $3 \mathrm{U} / \mathrm{mL}$ ) added at the $t_{90}$ time point on indirect twitches of the CBCNM. (d) The effects of $N$. atra venom $(3 \mu \mathrm{g} / \mathrm{mL})$ alone or with Aus pvAV $(3 \mathrm{U} / \mathrm{mL})$ added at the $\mathrm{t}_{90}$ time point on contractile responses to ACh $(1 \mathrm{mM}), \mathrm{CCh}(20 \mu \mathrm{M})$, and $\mathrm{KCl}(40 \mathrm{mM})$ in the CBCNM. ${ }^{*} p<0.05$, significantly different compared to control at $230 \mathrm{~min}$ $(\mathbf{a}, \mathbf{c})$ or compared to pre-venom response to same agonist $(\mathbf{b}, \mathbf{d}) .{ }^{\circledR} p<0.05$, significantly different compared to venom in the absence of antivenom at $230 \mathrm{~min}(\mathbf{a}, \mathbf{c})$ or compared to response to agonist in the absence of antivenom $(\mathbf{b}, \mathbf{d}), \mathrm{n}=5-6$.

The addition of Australian polyvalent antivenom, $(3 \mathrm{U} / \mathrm{mL}, 10 \times$ the recommended titre), at the $\mathrm{t}_{90}$ time point, after the addition of $N$. atra venom $(3 \mu \mathrm{g} / \mathrm{mL})$, partially restored the twitch height, i.e., reaching $35 \pm 4 \%(n=6)$ of the initial pre-venom twitch height (Figure $3 \mathrm{c})$. The addition of Australian polyvalent antivenom also significantly reversed the inhibition of responses to ACh and $\mathrm{CCh}$, while having no significant effect on the response to $\mathrm{KCl}$ (Figure 3d).

\subsection{In Vitro Myotoxicity}

$N$. atra venom $(30 \mu \mathrm{g} / \mathrm{mL})$ significantly inhibited twitches in the directly-stimulated chick biventer preparation, when compared to vehicle at $180 \mathrm{~min}(\mathrm{n}=5-6$; one-way ANOVA, $p<0.05$; Figure 4a). 
(a)

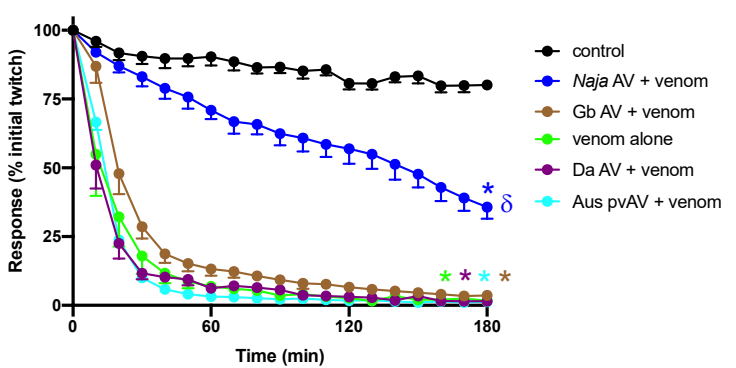

(c)

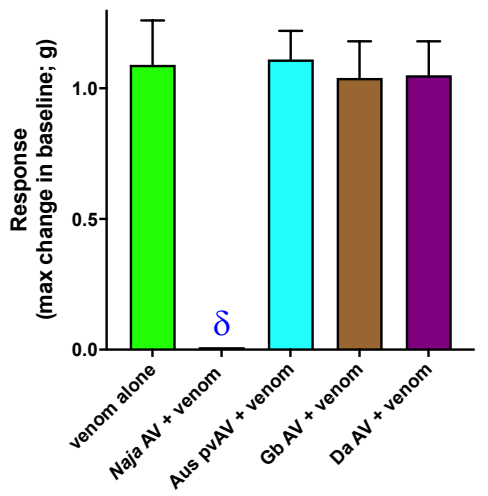

(b)

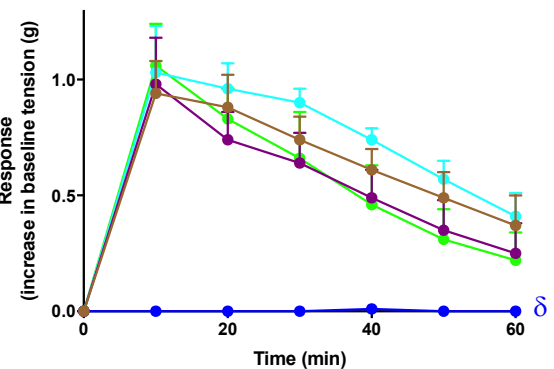

(d)

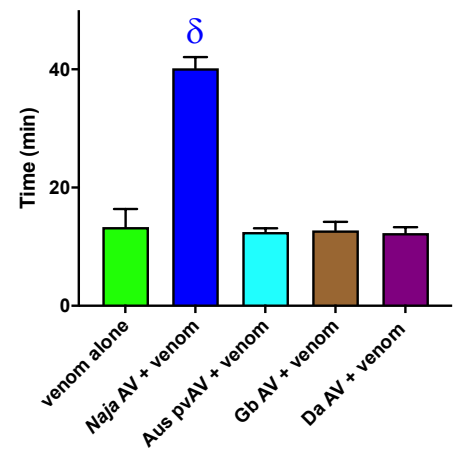

Figure 4. The myotoxic effects of Naja atra venom $(30 \mu \mathrm{g} / \mathrm{mL})$, in the presence and absence of different antivenoms, as indicated by the (a) change in twitch height in chick biventer cervicis preparation over $180 \mathrm{~min}$; (b) change in baseline tension of the chick biventer cervicis preparation over $60 \mathrm{~min}$; (c) $\max$ change in baseline gram tension achieved in $60 \mathrm{~min}$; and (d) the time achieve max change in baseline tension. ${ }^{*} p<0.05$, significantly different compared to control (a) at $180 \mathrm{~min} .{ }^{\delta} p<0.05$, significantly different compared to venom alone at $180 \mathrm{~min}$ (a) or $60 \mathrm{~min}$ (b) or compared to venom in the absence of antivenom (c,d). $\mathrm{n}=5-6$. Antivenoms were added $10 \mathrm{~min}$ prior to venom. N. atra antivenom (Naja AV); G. brevicaudus antivenom AV (Gb AV); D. acutus antivenom AV (Da AV); Australian polyvalent AV (Aus pvAV).

The prior addition of Chinese $N$. atra monovalent antivenom $200 \mu \mathrm{L}(4 \mathrm{U} / \mathrm{mL}, 1 \times$ the recommended titre) markedly attenuated, but did not prevent, twitch inhibition ( $\mathrm{n}=6$; one-way ANOVA, $p<0.05$; Figure $4 \mathrm{a})$ and abolished the venom-induced increase in baseline tension compared to venom $(30 \mu \mathrm{g} / \mathrm{mL})$ alone ( $n=5-6$; one-way ANOVA, $p<0.05$; Figure $4 b-d$ ), indicating partial attenuation of the myotoxic actions of $N$. atra venom.

In contrast, the prior addition of Australian polyvalent snake antivenom $200 \mu \mathrm{L}(33 \mathrm{U} / \mathrm{mL}$, $11 \times$ the recommended titre), G. brevicaudus antivenom $200 \mu \mathrm{L}(24 \mathrm{U} / \mathrm{mL}, 0.5 \sim 0.7 \times$ the recommended titre) or Chinese D. acutus monovalent antivenom $200 \mu \mathrm{L}(8 \mathrm{U} / \mathrm{mL}, 2 \sim 6 \times$ the recommended titre), failed to prevent or delay the venom-induced decrease in direct twitches $(n=5-6$; Figure $4 a)$ or venom-induced increase in baseline tension $(n=5-6$; Figure $4 b-d)$, indicating a lack of efficacy against the myotoxic actions of N. atra venom.

Control experiments (i.e., $200 \mu \mathrm{L}$ of each antivenom alone) indicated the antivenoms had no direct effect on tissue viability over a period of $180 \mathrm{~min}(\mathrm{n}=5-6$ for each antivenom).

\subsection{Cell Viability Assay}

\subsubsection{Venom Concentration-Response Curves}

Treatment of L6 cells with $N$. atra venom resulted in a concentration-dependent inhibition of cell viability (Figure 5), with an $\mathrm{IC}_{50}$ of $2.8 \pm 0.48 \mu \mathrm{g} / \mathrm{mL}$. 


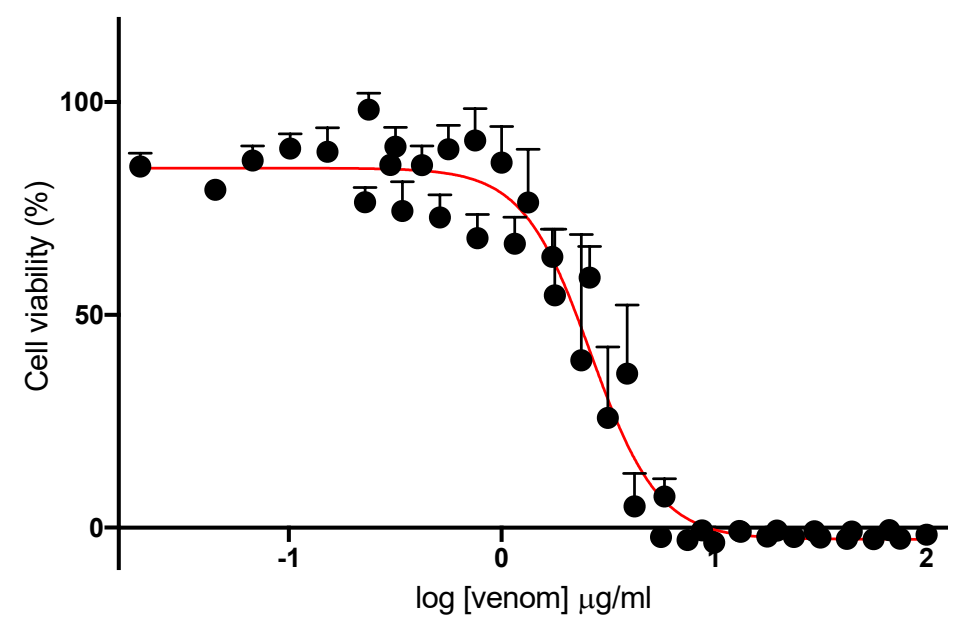

Figure 5. Concentration-dependent venom-induced inhibition of cell viability in L6 cells.

\subsubsection{Cell-Based Proliferation Assay-Efficacy of Antivenoms}

L6 cells were treated with $2 \%$ DMEM media supplemented with venom at concentrations of 0 , $2.5,5,10$, or $30 \mu \mathrm{g} / \mathrm{mL}$ and further supplemented with either no antivenom (i.e., venom alone) or with $N$. atra antivenom $(200 \mu \mathrm{L} ; 4 \mathrm{U} / \mathrm{mL})$, Australian polyvalent snake antivenom $(200 \mu \mathrm{L} ; 33 \mathrm{U} / \mathrm{mL})$, G. brevicaudus antivenom ( $200 \mu \mathrm{L} ; 24 \mathrm{U} / \mathrm{mL})$, or D. acutus monovalent antivenom $(200 \mu \mathrm{L} ; 8 \mathrm{U} / \mathrm{mL})$.

$N$. atra venom caused a significant decrease in cell viability at all concentrations examined when compared to cells treated with media alone $(p<0.05$; Figure 6). N. atra antivenom significantly attenuated the cytotoxic effect at all venom concentrations compared to control $(p<0.05$; Figure 6$)$. Australian polyvalent snake antivenom was less effective but still attenuated the cytotoxic effects at lower venom concentrations (i.e., $2.5-10 \mu \mathrm{g} / \mathrm{mL} ; p<0.05$; Figure 6). Neither G. brevicaudus antivenom or $D$. acutus antivenom were able to prevent the cytotoxicity at any venom concentration examined (Figure 6).

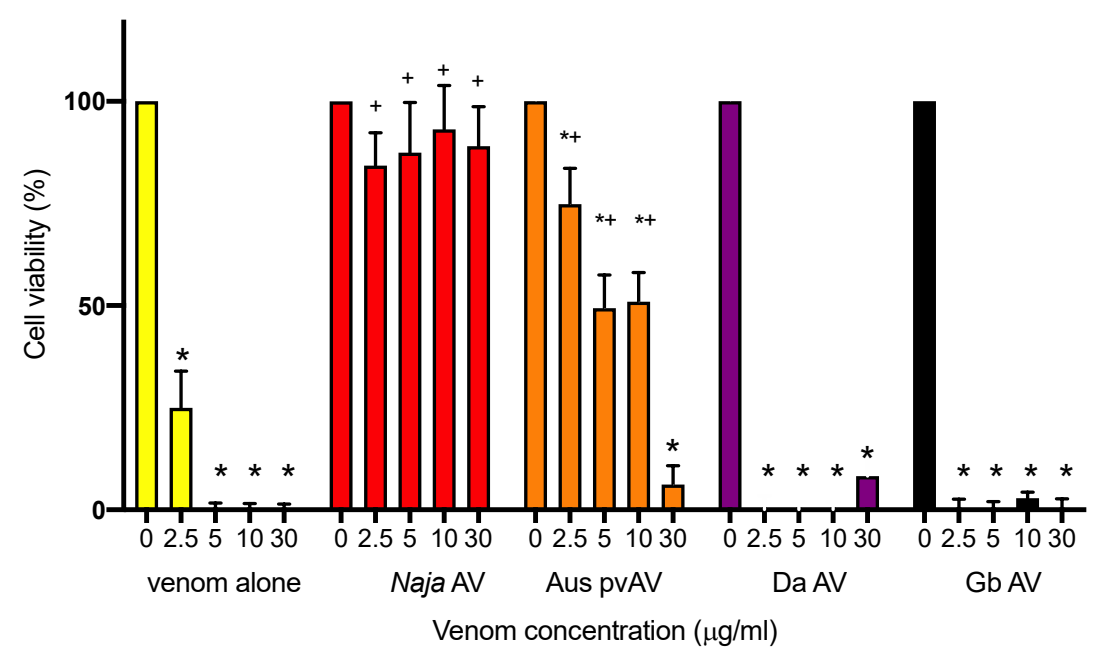

Figure 6. The effects of N. atra venom $(0-30 \mu \mathrm{g} / \mathrm{mL})$, in L6 cells, in the presence and absence of N. atra antivenom (Naja AV, $4 \mathrm{U} / \mathrm{mL}), \mathrm{G}$. brevicaudus antivenom $\mathrm{AV}(\mathrm{Gb} \mathrm{AV}, 24 \mathrm{U} / \mathrm{mL})$, D. acutus antivenom $\mathrm{AV}$ (Da AV, $8 \mathrm{U} / \mathrm{mL}$ ) or Australian polyvalent AV (Aus pvAV, $33 \mathrm{U} / \mathrm{mL}$ ). ${ }^{*} p<0.05$, significantly different from control (i.e., 0 venom); ${ }^{+} p<0.05$, significantly different from same concentration of venom in the absence of antivenom. 


\section{Discussion}

We have shown that $N$. atra venom from China displays potent in vitro neurotoxic, myotoxic and cytotoxic activity. The neurotoxic and cytotoxic effects of the venom were almost completely abolished by the prior addition of specific $N$. atra antivenom, whereas the myotoxic effects were only partially prevented. Interestingly, an Australian polyvalent antivenom, which is raised against the venoms from a range of Australian elapids (i.e., Acanthophis antarcticus, Notechis scutatus, Oxyuranus scutellatus, Pseudechis australis, and Pseudonaja textilis) and does not contain specific antibodies against $N$. atra venom, displayed similar activity against the neurotoxic effects of $N$. atra venom but was less effective against the cytotoxic effects and ineffective against the myotoxic effects. The Australian polyvalent antivenom was included in our study as the venoms of the Australian elapids contain a range of post-synaptic, pre-synaptic, and myotoxic components. These components are likely to have close structural similarities with some of the components in the venom of the Chinese cobra given we have previously shown that Australian Tiger snake (N. scutatus) antivenom prevents the in vitro neurotoxicity induced by N. haje (Egyptian cobra) venom [12], and Australian polyvalent snake antivenom prevents the in vitro neurotoxicity induced by N. kaouthia (monocled cobra) venom [13].

The two Chinese monovalent viper antivenoms (i.e., G. brevicaudus antivenom and D. acutus antivenom antivenom) had no efficacy against the myotoxic or cytotoxic effects of $N$. atra venom. We did not examine the efficacy of the Chinese viper antivenoms against the neurotoxic effects of $N$. atra venom as this is not a clinical outcome of envenoming by this species, and the antivenoms are used in China to treat the myotoxic symptoms of N. atra envenoming. Four monovalent snake antivenoms are available in mainland China, and cross-neutralization by using nonspecific antivenoms for snakebite is recommended in the Chinese 2018 Expert Consensus on snakebites [2]. However, it appears as though the two viper antivenoms have no efficacy against $N$. atra venom.

We used the chick biventer cervicis nerve-muscle preparation, which contains both focally- and multiply-innervated skeletal muscle fibers, to examine neurotoxicity and myotoxicity. This preparation enables the determination of the site of action of venoms/toxins, i.e., either at the pre-synaptic nerve terminal, post-synaptic nerve terminal or underlying skeletal muscle $[14,15]$. The time taken to cause $90 \%$ (i.e., $t_{90}$ ) inhibition of nerve-mediated twitches can be used to compare the neurotoxic potency of venoms/toxins. N. atra venom abolished indirect twitches in a time-dependent and concentration-dependent manner, as well as inhibiting contractile responses to exogenous $\mathrm{ACh}$ and $\mathrm{CCh}$, while reducing responses to $\mathrm{KCl}$, indicating that it acts post-synaptically and has myotoxic effects on the tissue. The Chinese N. atra antivenom was highly efficacious when added prior to venom and was also able to partially reverse the inhibitory effects of the venom when added at the $t_{90}$ time point. The failure to fully reverse the decline in twitch height is likely to be due to a number of factors including the contribution of myotoxins and the lack of reversibility of some neurotoxins. Indeed, we have previously shown that the short-chain post-synaptic neurotoxin $\alpha$-Elapitoxin-Na1a, which accounts for approximately $9 \%$ of $N$. atra venom, displays pseudo-irreversible antagonism at the skeletal muscle nicotinic acetylcholine receptor and is only partially reversed by antivenom [7]. Interesting, the Australian polyvalent snake venom displayed similar efficacy against the neurotoxic effects of the venom indicating that the antigenic components in this antivenom, which is raised against a number of venoms from Australian elapid snakes containing postsynaptic and/or presynaptic neurotoxins, are able to recognize the neurotoxic components of $N$. atra venom.

Despite possible geographical differences in venom composition, the percentage of cardiotoxins and neurotoxins reported in N. atra venom ranges from $52-68 \%$ and $11-23 \%$, respectively $[3,4,6]$. Cardiotoxins, which target cell membranes, are likely to be the main components contributing to the soft tissue necrosis and myotoxicity [16-20]. Indeed, N. atra venom has been shown to display high levels of cytotoxicity [21]. Although, as indicated above, N. atra venom is highly neurotoxic in vitro, it is only mildly neurotoxic in humans. This is most likely due to the neurotoxic components being short-chain neurotoxins which readily dissociate from human muscle nAChRs [7]. Interestingly, a recent study found that the post-synaptic $\alpha$-neurotoxins in $N$. atra venom bind to the alpha- $1 \mathrm{nAChR}$ orthosteric site 
with selectivity towards the amphibian mimotope over lizard, avian and rodent mimotopes indicative of prey selectivity [22]. Despite the early usage and administration of large doses of N. atra antivenom in envenomed patients, severe wound necrosis or chronic necrotic ulceration causing extensive local tissue injuries are commonly reported [9-11]. Although subsequent wound infection due to heavy bacterial load introduced by the fangs $[23,24]$ might contribute to this clinical dilemma, there is the possibility of a lack of efficacy of the specific antivenom against the myotoxic effect of the venom.

As N. atra venom significantly inhibited responses to $\mathrm{KCl}$ in the indirectly stimulated chick biventer experiments, the presence of myotoxic activity in the venom was further examined in the directly stimulated chick biventer preparation. N. atra venom abolished direct twitches and induced an increase in baseline tension indicative of myotoxicity [14,15]. Prior addition of N. atra antivenom delayed, but did not prevent, inhibition of direct twitches, but abolished the increase in baseline tension. However, the inability of $N$. atra antivenom to fully prevent myotoxicity may not indicate a lack of full efficacy of the antivenom. Given that the myotoxic effects were studied at $10 \times$ the concentration at which the neurotoxic effects were studied (i.e., $30 \mu \mathrm{g} / \mathrm{mL}$ compared to $3 \mu \mathrm{g} / \mathrm{mL}$ ), a ratio which is in line with many of our previous neurotoxic/myotoxic studies [25-27], it is likely that increasing the antivenom concentration, e.g., at least double the manufacturer's recommended amount, may full prevent the myotoxic effects. Indeed, we needed to add $2 \times$ the manufacturer's recommendation to abolish venom neurotoxicity. However, we limited the maximum amount of antivenom used in the myotoxicity study given that excessive amounts of antivenom can alter the osmolarity of the physiological salt solution in the organ bath and affect tissue viability. The Australian polyvalent snake antivenom and the two Chinese monovalent viper antivenoms failed to significantly inhibit either the decrease in twitch height or increase in baseline tension.

$N$. atra antivenom also neutralized the potent cytotoxic effects of $N$. atra venom in L6 rat skeletal muscle cells. It is worth noting that the antivenom was protective against $30 \mu \mathrm{g} / \mathrm{mL}$ of venom in this assay, further supporting that the lack of full efficacy in the chick biventer myotoxic study was due to an insufficient concentration of antivenom. This problem did not occur in the cell assay given the much lower volumes used. The Australian polyvalent snake antivenom was protective at lower concentrations of venom in the cytotoxicity assay, whereas the two viper antivenoms had no significant protective effect. Although the venoms from these Chinese viper species (i.e., G. brevicaudus and D. acutus) can cause local tissue swelling and necrosis in envenomed humans, their venom proteomes and the relative abundance of major components are quite different to the Chinese elapid N. atra venom [3,5]. Therefore, it is not surprising that these antivenoms were unable to prevent the myotoxicity and cytotoxicity induced by N. atra venom. Our results strongly suggest that these viper antivenoms are unlikely to neutralize the effects of venom in patients envenomed by N. atra. Interestingly, the Australian polyvalent snake antivenom, which is raised against the venoms from five species of highly venomous terrestrial Australian elapids, failed to prevent $N$. atra venom induced myotoxicity in vitro while showing capability of fully preventing and even partially reversing N. atra venom induced neurotoxicity and cytotoxicity. This divergence has not been previously reported and could be explained by further venomic comparison studies between the species in the future.

\section{Conclusions}

In summary, we have, for the first time, examined the in vitro neurotoxic, myotoxic, and cytotoxic effects of $N$. atra venom and the ability of specific Chinese N. atra monovalent antivenom, non-specific Australian polyvalent snake antivenom, and Chinese G. brevicaudus monovalent antivenom and Chinese $D$. acutus monovalent antivenom to neutralize these effects. Our studies indicate that Chinese N. atra venom causes potent in vitro neurotoxicity, myotoxicity, and cytotoxicity, which is, largely, neutralized by N. atra antivenom. While the Australian polyvalent antivenom was equally efficacious against the neurotoxic effects, indicating the presence of similar antigenic neurotoxins, it was ineffective against the myotoxicity and only partially protective against the cytotoxic effects. The Chinese viper 
antivenoms were ineffective and do not appear to display any cross-reactivity against the myotoxic and cytotoxic components of N. atra venom.

Author Contributions: Conceptualization, Q.L., G.K.I. and W.C.H.; methodology, Q.L., T.M.H., N.K. and W.C.H.; formal analysis, Q.L., N.K. and W.C.H.; investigation, Q.L., N.K.; data curation and writing-original draft preparation, Q.L., N.K. and W.C.H.; writing-review and editing and supervision, G.K.I. and W.C.H.; funding acquisition, Q.L., G.K.I. and W.C.H.; project administration, G.K.I. and W.C.H. All authors have read and agreed to the published version of the manuscript.

Funding: This study was supported by an Australian National Health and Medical Research Council (NHMRC) Senior Research Fellowship (ID: 1061041) awarded to G.K.I., a NHMRC Centers for Research Excellence Grant (ID:1110343) awarded to G.K.I. and W.C.H., and a 2016 Guangzhou Municipal University scientific research project Grant (ID: 1201620144) awarded to Q.L.

Conflicts of Interest: The authors declare no conflict of interest. The funders had no role in the design of the study; in the collection, analyses, or interpretation of data; in the writing of the manuscript, or in the decision to publish the results.

\section{References}

1. Em, Z. Snakes in China, 1st ed.; Anhui Science and Technology Publishing House: Hefei, China, 2006; pp. $4,347,349$.

2. Experts Group of Snake-Bites Rescue and Treatment Consensus in China. Expert consensus on China snake-bites rescue and treatment. Chin. J. Emerg. Med. 2018, 27, 1315-1322. [CrossRef]

3. Li, S.; Wang, J.; Zhang, X.; Ren, Y.; Wang, N.; Zhao, K.; Chen, X.; Zhao, C.; Li, X.; Shao, J.; et al. Proteomic characterization of two snake venoms: Naja naja atra and Agkistrodon halys. Biochem. J. 2004, 384, 119-127. [CrossRef] [PubMed]

4. Huang, H.W.; Liu, B.S.; Chien, K.Y.; Chiang, L.C.; Huang, S.Y.; Sung, W.C.; Wu, W.G. Cobra venom proteome and glycome determined from individual snakes of Naja atra reveal medically important dynamic range and systematic geographic variation. J. Proteom. 2015, 128, 92-104. [CrossRef]

5. Liu, C.C.; Lin, C.C.; Hsiao, Y.C.; Wang, P.J.; Yu, J.S. Proteomic characterization of six Taiwanese snake venoms: Identification of species-specific proteins and development of a SISCAPA-MRM assay for cobra venom factors. J. Proteom. 2018, 187, 59-68. [CrossRef]

6. Shan, L.L.; Gao, J.F.; Zhang, Y.X.; Shen, S.S.; He, Y.; Wang, J.; Ma, X.M.; Ji, X. Proteomic characterization and comparison of venoms from two elapid snakes (Bungarus multicinctus and Naja atra) from China. J. Proteom. 2016, 138, 83-94. [CrossRef] [PubMed]

7. Liang, Q.; Huynh, T.M.; Isbister, G.K.; Hodgson, W.C. Isolation and pharmacological characterization of $\alpha$-Elapitoxin-Na1a, a novel short-chain postsynaptic neurotoxin from the venom of the Chinese Cobra (Naja atra). Biochem. Pharmacol. 2020. [CrossRef]

8. Silva, A.; Cristofori-Armstrong, B.; Rash, L.D.; Hodgson, W.C.; Isbister, G.K. Defining the role of post-synaptic alpha-neurotoxins in paralysis due to snake envenoming in humans. Cell Mol. Life Sci. 2018, 75, 4465-4478. [CrossRef]

9. Wang, W.; Chen, Q.F.; Yin, R.X.; Zhu, J.J.; Li, Q.B.; Chang, H.H.; Wu, Y.B.; Michelson, E. Clinical features and treatment experience: A review of 292 Chinese cobra snakebites. Environ. Toxicol. Pharmacol. 2014, 37, 648-655. [CrossRef]

10. Mao, Y.C.; Liu, P.Y.; Chiang, L.C.; Lai, C.S.; Lai, K.L.; Ho, C.H.; Wang, T.H.; Yang, C.C. Naja atra snakebite in Taiwan. Clin. Toxicol. (Philadelphia) 2018, 56, 273-280. [CrossRef]

11. Wong, O.F.; Lam, T.S.; Fung, H.T.; Choy, C.H. Five-year experience with Chinese cobra (Naja atra)-related injuries in two acute hospitals in Hong Kong. Hong Kong Med. J. 2010, 16, 36-43.

12. Kornhauser, R.; Isbister, G.K.; O’Leary, M.A.; Mirtschin, P.; Dunstan, N.; Hodgson, W.C. Cross-neutralisation of the neurotoxic effects of Egyptian Cobra venom with commercial Tiger snake antivenom. Basic Clin. Pharmacol. Toxicol. 2013, 112, 138-143. [CrossRef] [PubMed]

13. Silva, A.; Hodgson, W.C.; Isbister, G.K. Cross-neutralisation of in vitro neurotoxicity of Asian and Australian snake neurotoxins and venoms by different antivenoms. Toxins 2016, 8, 302. [CrossRef] [PubMed]

14. Harvey, A.L.; Barfaraz, A.; Thomson, E.; Faiz, A.; Preston, S.; Harris, J.B. Screening of snake venoms for neurotoxic and myotoxic effects using simple in vitro preparations from rodents and chicks. Toxicon 1994, 32, 257-265. [CrossRef] 
15. Hodgson, W.C.; Wickramaratna, J.C. In vitro neuromuscular activity of snake venoms. Clin. Exp. Pharmacol. Physiol. 2002, 29, 807-814. [CrossRef] [PubMed]

16. Chang, C.C.; Chuang, S.T.; Lee, C.Y.; Wei, J.W. Role of cardiotoxin and phospholipase A in the blockade of nerve conduction and depolarization of skeletal muscle induced by cobra venom. Br. J. Pharmacol. 1972, 44, 752-764. [CrossRef] [PubMed]

17. Ownby, C.L.; Fletcher, J.E.; Colberg, T.R. Cardiotoxin 1 from cobra (Naja naja atra) venom causes necrosis of skeletal muscle in vivo. Toxicon 1993, 31, 697-709. [CrossRef]

18. Huang, S.J.; Kwan, C.Y. Inhibition by multivalent cations of contraction induced by Chinese cobra venom cardiotoxin in guinea pig papillary muscle. Life Sci. 1996, 59, P155-PI60. [CrossRef]

19. Vignaud, A.; Hourdé, C.; Butler-Browne, G.; Ferry, A. Differential recovery of neuromuscular function after nerve/muscle injury induced by crude venom from Notechis scutatus, cardiotoxin from Naja atra and bupivacaine treatments in mice. Neurosci. Res. 2007, 58, 317-323. [CrossRef]

20. Wang, C.H.; Monette, R.; Lee, S.C.; Morley, P.; Wu, W.G. Cobra cardiotoxin-induced cell death in fetal rat cardiomyocytes and cortical neurons: Different pathway but similar cell surface target. Toxicon 2005, 46, 430-440. [CrossRef]

21. Panagides, N.; Jackson, T.N.W.; Ikonomopoulou, M.P.; Arbuckle, K.; Pretzler, R.; Yang, D.C.; Ali, S.A.; Koludarov, I.; Dobson, J. How the cobra got its flesh-eating venom: Cytotoxicity as a defensive innovation and its co-evolution with hooding, aposematic marking and spitting. Toxins 2017, 9, 103. [CrossRef]

22. Harris, R.J.; Zdenek, C.N.; Harrich, D.; Frank, N.; Fry, B.G. An appetite for destruction: Detecting prey-selective binding of $\alpha$-neurotoxins in the venom of Afro-Asian elapids. Toxins 2020, 12, 205. [CrossRef] [PubMed]

23. Lam, K.K.; Crow, P.; Ng, K.H.; Shek, K.C.; Fung, H.T.; Ades, G.; Grioni, A.; Tan, K.S.; Yip, K.T.; Lung, D.C.; et al. A cross-sectional survey of snake oral bacterial flora from Hong Kong, SAR, China. Emerg. Med. J. 2011, 28, 107-114. [CrossRef] [PubMed]

24. Mao, Y.C.; Liu, P.Y.; Hung, D.Z.; Lai, W.C.; Huang, S.T.; Hung, Y.M.; Yang, C.C. Bacteriology of Naja atra snakebite wound and its implications for antibiotic therapy. Am. J. Trop. Med. Hyg. 2016, 94, 1129-1135. [CrossRef] [PubMed]

25. Lumsden, N.G.; Ventura, S.; Dauer, R.; Hodgson, W.C. A biochemical and pharmacological examination of Rhamphiophis oxyrhynchus (Rufous beaked snake) venom. Toxicon 2005, 45, 219-231. [CrossRef] [PubMed]

26. Ramasamy, S.; Isbister, G.K.; Hodgson, W.C. The efficacy of two antivenoms against the in vitro myotoxic effects of black snake (Pseudechis) venoms in the chick biventer cervicis nerve-muscle preparation. Toxicon 2004, 44, 837-845. [CrossRef] [PubMed]

27. Wickramaratna, J.C.; Hodgson, W.C. A pharmacological examination of venoms from three species of death adder (Acanthopis antarcticus, Acanthopis praelongus and Acanthopis pyrrhus). Toxicon 2001, 39, $209-216$. [CrossRef]

(C) 2020 by the authors. Licensee MDPI, Basel, Switzerland. This article is an open access article distributed under the terms and conditions of the Creative Commons Attribution (CC BY) license (http://creativecommons.org/licenses/by/4.0/). 